\title{
Bridging a yawning chasm: EEG investigations into the debate concerning the role of the human mirror neuron system in contagious yawning
}

\author{
Nicholas R. Cooper • Ignazio Puzzo • Adam D. Pawley • \\ Ruby A. Bowes-Mulligan • Emma V. Kirkpatrick • \\ Pavlina A. Antoniou - Steffan Kennett
}

Published online: 24 December 2011

(C) Psychonomic Society, Inc. 2011

\begin{abstract}
Ongoing debate in the literature concerns whether there is a link between contagious yawning and the human mirror neuron system (hMNS). One way of examining this issue is with the use of the electroencephalogram (EEG) to measure changes in mu activation during the observation of yawns. $\mathrm{Mu}$ oscillations are seen in the alpha bandwidth of the EEG $(8-12 \mathrm{~Hz})$ over sensorimotor areas. Previous work has shown that mu suppression is a useful index of hMNS activation and is sensitive to individual differences in empathy. In two experiments, we presented participants with videos of either people yawning or control stimuli. We found greater mu suppression for yawns than for controls over right motor and premotor areas, particularly for those scoring higher on traits of empathy. In a third experiment, auditory recordings of yawns were compared against electronically scrambled versions of the same yawns. We observed greater mu suppression for yawns than for the controls over right lateral premotor areas. Again, these findings were driven by those scoring highly on empathy. The results from these
\end{abstract}

\footnotetext{
N. R. Cooper · I. Puzzo • A. D. Pawley • R. A. Bowes-Mulligan P. A. Antoniou $\cdot$ S. Kennett

University of Essex,

Colchester, UK

I. Puzzo

University of Reading,

Reading, UK

E. V. Kirkpatrick

Newcastle University,

Newcastle upon Tyne, UK

N. R. Cooper $(\bowtie)$

Centre for Brain Science, Department of Psychology,

University of Essex,

Colchester CO4 3SQ Essex, UK

e-mail: ncooper@essex.ac.uk
}

experiments support the notion that the hMNS is involved in contagious yawning, emphasise the link between contagious yawning and empathy, and stress the importance of good control stimuli.

Keywords Yawn - Contagious yawning · Contagion - EEG . Alpha $\cdot \mathrm{Mu} \cdot$ Empathy

These are interesting times for a field concerned with a physiological process often associated with boredom, namely yawning. In particular, the study of contagious yawning appears to offer a fruitful avenue of investigation for the growing fields of developmental, affective, and social neuroscience. Contagious yawning refers to the phenomenon wherein seeing or hearing someone yawn, or even reading or thinking about yawning, can trigger a yawn in the beholder (Platek, Mohamed, \& Gallup, 2005). It typically occurs in $40 \%-60 \%$ of the population (Platek, Critton, Myers, \& Gallup, 2003; Provine, 1989), which begs the question, what underlies the individual differences in this phenomenon? To date, much of the evidence points to a link between contagious yawning and the level of empathy of the individual (Platek, 2010; Platek et al., 2003; Platek et al., 2005; Schürmann et al., 2005; Senju et al., 2007). Indeed, clinical populations who typically exhibit impairments in empathic processing (e.g., schizophrenia and the autism spectrum disorders, or ASD) also demonstrate a paucity of contagious yawning under normal circumstances (Haker \& Rossler, 2009; Senju et al., 2007), but in the case of ASD, this can be rectified given instructions to fixate on the eyes of the person yawning (Senju et al., 2009).

One of the main candidate mechanisms for empathic processing in general is the mirror neuron system. Mirror neurons were originally observed in monkeys and are a 
specific type of motor cell that fires not only when the animal makes a specific movement, but also when it observes the same movement being carried out (di Pellegrino, Fadiga, Fogassi, Gallese, \& Rizzolatti, 1992; Gallese, Fadiga, Fogassi, \& Rizzolatti, 1996). Since these original observations, a multitude of studies have examined human correlates of such activation using indirect methods such as fMRI, electroencephalograms (EEGs), or transcranial magnetic stimulation, and these studies have predominantly shown that such a mechanism (often referred to as the human mirror neuron system; hMNS) exists in humans. A recent study using single-cell recording in humans claims to have found the first direct evidence for the existence of mirror neurons per se in humans (Mukamel, Ekstrom, Kaplan, Iacoboni, \& Fried, 2010). It has been postulated that mirror neurons may underlie many social skills, such as action understanding, imitation, theory of mind, language, and empathy (Rizzolatti \& Craighero, 2004). With regard to empathy, several studies have demonstrated a correlation between it and hMNS activation. For instance, Kaplan and Iacoboni (2006) presented hand stimuli in various conditions designed to contrast intentional aspects of the scene and observed BOLD activation in the right inferior hMNS that correlated with empathic concern on the Interpersonal Reactivity Index (IRI; Davis, 1983). Using an auditory paradigm, Gazzola, Aziz-Zadeh, and Keysers (2006) found a correlation between hMNS activation to the sounds of actions and the Perspective Taking subscale of the IRI, and when observing and imitating emotional facial expressions, Pfeifer, Iacoboni, Mazziotta, and Dapretto (2008) found that frontal hMNS activity correlated with both empathic behaviour and interpersonal skills. It has also been hypothesised that a faulty hMNS may underlie many of the social deficits (including empathy) observed in ASD (Martineau, Andersson, Barthélémy, Cottier, \& Destrieux, 2010; Oberman et al., 2005; Ramachandran \& Oberman, 2006) and schizophrenia (Enticott et al., 2008), and may account for the individual differences in autistic traits observed in the general population (Puzzo, Cooper, Vetter, \& Russo, 2010).

Given this putative link between empathy and the hMNS and the deficits in both contagious yawning and empathic skills observed in ASD and schizophrenia, it would not appear unreasonable to speculate that the hMNS may indeed be involved in contagious yawning (Cooper, Puzzo, \& Pawley, 2008). However, the neuroimaging evidence to date is less than convincing, and consequently there is disagreement in the literature as to whether or not the hMNS is an important factor in contagious yawning. Generally, neuroimaging research on contagious yawning depends crucially on the design of the control conditions. For instance, Platek et al. (2005), when comparing fMRI BOLD signals between participants observing yawns or laughs, observed unique activation to yawns in the precuneus and posterior cingulate areas associated with empathic processing, but which are not part of the hMNS. However, given the socially contagious nature of laughter, the use of laughs as a control stimulus may have masked any contribution of the hMNS to contagious yawns. Schürmann et al. (2005) using a video of a "nonnameable mouth-andtongue" action as a control condition, found activation to yawns in the right posterior superior temporal sulcus (STS) and bilaterally in the anterior STS, but not in frontal hMNS areas. The authors proposed that this indicates that contagious yawning does not require the detailed action understanding afforded by the hMNS. However, it should be noted that STS is considered by some to be a part of the extended mirror neuron system, although not a core area (Pineda, 2008), since it contains cells that are involved in coding biological motion (Jellema, Baker, Oram, \& Perrett, 2002). More recently, Nahab and colleagues used fMRI to examine reactivity to yawn stimuli in comparison to three control stimuli: a still face, a cough, and a gape (Nahab, 2010; Nahab, Hattori, Saad, \& Hallett, 2009). Unique activation to yawns was observed in the ventromedial prefrontal cortex, which was positively correlated with the urge to yawn; activation common to all stimuli was noted in hMNS areas. The only neuroimaging study to date to find evidence of specific hMNS involvement in contagious yawning has come from Arnott, Singhal, and Goodale (2009). Using an auditory paradigm, they contrasted the sound of yawns with electronically scrambled versions of the same stimuli. In this context, greater BOLD activation to yawns was observed in the right inferior frontal gyrus (a core area of the hMNS), and this activation was greatest for stimuli associated with high ratings for an urge to yawn.

Consequently, we undertook a series of experiments in an attempt to address the discrepancies between these neuroimaging investigations of contagious yawning. The EEG was our psychophysiological tool of choice, as it affords both a much higher temporal resolution than fMRI, as well as a readily identifiable index of hMNS activation-namely, mu suppression. $M u$ suppression (or mu event-related desynchronisation, ERD) refers to a decrease in power in the alpha $(8-12 \mathrm{~Hz})$ and sometimes the lower beta $(12-20 \mathrm{~Hz})$ bandwidths of the EEG over sensorimotor areas relative to a reference interval; an increase in mu power is referred to as event-related synchronisation, or ERS. In this article, we will use the terms mu suppression and alpha ERD (over sensorimotor areas) interchangeably. ERD is observed during motor acts (Arroyo et al., 1993; Chatrian, Petersen, \& Lazarte, 1959; Gastaut, 1952), during preparation for action (Jasper \& Penfield, 1949), while imagining a movement (Pfurtscheller, Neuper, Brunner, \& da Silva, 2005), and, pertinent to the present study, while observing a movement (Gastaut \& Bert, 1954; Hari et al., 1998; Kilner, Marchant, \& Frith, 2009; Muthukumaraswamy \& Johnson, 2004; Pineda, 2005; Streltsova, Berchio, Gallese, \& Umiltà, 2010). As a result, $\mathrm{mu}$ suppression has been posited to be a useful indicator of 
action observation pattern matching in the cortex, and at present, the best candidate area for this process appears to be the hMNS. Indeed, mu suppression to various hand movements has been shown to closely mirror BOLD activation in areas analogous in humans to the mirror neuron areas in primate studies (Perry \& Bentin, 2009); to be modulated by the laterality of the presentation stimulus, consistent with the reactivity of mirror neurons in area F5 in monkeys (Kilner et al., 2009); and to be dynamically modulated similarly in both action observation and action performance (Press, Cook, Blakemore, \& Kilner, 2011). Consequently, mu suppression during action observation is usually interpreted as an index of activity in the hMNS (Kilner et al., 2009; Pineda, 2005, 2008). Indeed, whereas until recently, mu suppression during action observation has been postulated to result from postsynaptic modulation from mirror neurons in premotor cortex (Pineda, 2008; Rizzolatti \& Craighero, 2004), recent evidence for socalled $M 1$ view cells in primary motor cortex with mirrorneuron-like properties (Dushanova \& Donoghue, 2010) suggests that perhaps mu suppression may be a more direct measure of hMNS than was previously believed, as M1 may itself be a part of the mirror neuron system (Press et al., 2011).

Given the proposed multimodal nature of hMNS activity, we decided to examine the possible link between it and contagious yawning using both visual and auditory protocols. In Experiments 1 and 2, we used visually presented videos of yawns and gapes. Then, Experiment 3 was a constructive replication of Arnott et al. (2009) using auditory stimuli. Given Arnott et al.'s findings of right inferior frontal hMNS activation during yawns, we focused our analyses on analogous areas (i.e., the right $\mathrm{FC}$ and $\mathrm{C}$ electrode strips). We hypothesised that yawn stimuli would elicit greater mu suppression than would control (non-yawn) stimuli. We were also interested in the possible links between empathy, contagious yawning, and mirror neurons, and so we also hypothesised that mu suppression would be greater for those scoring high on a measure of empathy (the IRI) and that this effect would be greater during yawns than during nonyawns.

\section{Experiment 1}

Method

Participants A total of 79 volunteers completed the IRI (Davis, 1983). Their mean score was $65.68(S D=10.97)$. From these empathy scores, two experimental groups were derived: one group representing high scores $(>1$ standard deviation above the mean; high-empathy group) and one group representing low scores $(>1$ standard deviation below the mean; low-empathy group). A group of 10 participants were assigned to the high-empathy group, and 9 were in the low-empathy group. Thus, 19 participants (14 female), with a mean age of 22 years $(S D=5)$, took part in the EEG phase of the study. Two of the participants were excluded for excessively noisy data (one from each empathy group), resulting in the data from 17 participants being analysed. Participants gave written informed consent, and the study was approved by the University of Essex Ethics Committee.

Materials Empathy levels were measured using the IRI (Davis, 1983), which consists of four 7-item subscales, all thought to measure different facets of empathy. These are the Perspective Taking, Empathic Concern, Personal Distress, and Fantasy subscales. The experimental stimuli were 3-s long video clips of people either yawning or gaping (opening and closing their mouths to the same time scale as the yawns), with the soundtrack removed. A 3-s presentation time was chosen because it had previously been shown to produce robust findings with regard to mu suppression, as it is long enough to show the effect, but short enough to allow for many trial repetitions, thereby improving the signal-to-noise ratio (Puzzo, Cooper, Cantarella, \& Russo, 2011). Consequently, videos of yawns were trimmed to $3,000 \mathrm{~ms}$, removing the initial and final stages of the yawn while keeping the opening of the mouth, maximum stretch, and partial closing of the mouth. There were 10 different stimuli (5 yawns and 5 gapes) filmed using five different actors. Each video clip was repeated five times, amounting to 50 trials in total, presented in a randomised order. Each trial consisted of the presentation of (1) a fixation cross $(2,000 \mathrm{~ms}$; $20 \times 25 \mathrm{~mm}),(2)$ the stimulus video $(3,000 \mathrm{~ms} ; 300 \times$ $180 \mathrm{~mm})$, and (3) a blank screen $(5,000 \mathrm{~ms})$; thus, each trial lasted $10 \mathrm{~s}$. The stimuli were presented using Superlab software (Cedrus Corporation, San Pedro, CA) on an Apple PowerMac (2-GHz Power PC G5; Apple Inc., Cupertino, CA).

In order to ensure that the videos used in this experiment actually elicited contagious yawning, they were tested on 28 naïve participants ( 15 female; mean age $=25$ years) who did not take part in any of the other experiments reported in this article. The participants were presented with a block of yawn videos followed by a block of gape videos, or vice versa (presentation order was counterbalanced across participants). Within each block, each video was repeated three times in a random sequence. The results were as follows: More people yawned whilst viewing the yawn videos (15) than during the gaping videos $\left[4 ; \chi^{2}(1, N=56)=9.64, p=.002\right]$. More people felt an urge to yawn whilst viewing the yawn videos (24) than during the gaping videos $\left[10 ; \chi^{2}(1, N=56)=\right.$ $9.64, p=.002]$. The number of yawns elicited by the stimuli was greater whilst viewing the yawn videos (26) than during the gaping videos $[5 ; F(1,26)=16.82, p<.001]$. The number of urges to yawn elicited by the stimuli was greater whilst viewing the yawn videos (91) than during the gaping videos $[25 ; F(1,26)=26.97, p<.001]$. Finally, there were no 
interactions with presentation order (i.e., yawn video first vs. gape video first; $p \mathrm{~s}>.5$ ).

Procedure The participants were seated in a darkened, quiet room, $70 \mathrm{~cm}$ in front of a 19-in. computer monitor (Viglen Ltd., St. Albans, UK). EEGs were recorded while the participants first completed an eye-movement calibration protocol (Croft \& Barry, 2000), followed by 2 min rest with eyes closed, followed by the yawn-gape protocol. In order to ensure that attention was sustained whilst watching the video clips, participants were instructed to count silently, to themselves, the number of times they saw a certain type of clip (e.g., count how many male yawns). The type of stimulus to be counted was counterbalanced across participants.

EEG data acquisition The EEG data were recorded with Neuroscan 4.3.1 acquisition software and SynampsII amplifiers from 21 scalp sites (FC5, FC3, FC1, FCz, FC2, FC4, FC6, C5, C3, C1, Cz, C2, C4, C6, CP5, CP3, CP1, CPz, CP2, CP4, and CP6) using a Quick-Cap arranged according to the international 10-10 system (Compumedics, Melbourne, Australia). Eye movements were recorded using four facial electrodes - above and below the left eye and on the outer canthi of the eyes. Impedances for all of the electrodes were reduced to below $10 \mathrm{k} \Omega$ before the start of each session. All of the data were continuously sampled at $1,000 \mathrm{~Hz}$, with a bandpass filter of $0.1-200 \mathrm{~Hz}$ and a $50-\mathrm{Hz}$ notch filter. Online, EEG data were referenced to a point midway between $\mathrm{Cz}$ and $\mathrm{CPz}$ and grounded midway between Fz and FPz.

EEG data preparation Following visual inspection of the data, noisy data blocks were rejected. This included periods with noise artefacts caused by the participants themselves yawning; consequently, no data containing yawns from the participants were included in the analysis. Similarly, segments in which participants attempted to suppress yawns (causing excess noise due to jaw or teeth clenching) were also excluded from analysis. Bad electrodes were excluded on a participant-by-participant basis. Ocular artefact rejection was carried out using methods described earlier by Croft and Barry (2000). All of the data were re-referenced to a common average reference, and a second automatic artefact rejection sweep was carried out, with exclusion parameters set at $\pm 75 \mathrm{mV}$. In order to calculate ERD/ERS, the data were epoched from $-1,000$ to $3,250 \mathrm{~ms}$ relative to the start of each video clip, and the following steps were performed using the event-related band-power transform in Neuroscan Edit 4.3 (Compumedics, Melbourne, Australia): The data underwent complex demodulation and concurrent filtering (zero-phase-shift, 24-dB roll-off envelope computed) into low alpha $(8-10 \mathrm{~Hz})$ and upper alpha $(10-12 \mathrm{~Hz})$ bands; these were trimmed $(500 \mathrm{~ms}$ from each end, to remove filter warm-up artefacts) and averaged. A reference interval of -500 to $0 \mathrm{~ms}$ was used to calculate the percentage change of two active periods (early [0-1,000 ms] and late [1,000-2,500 ms]) from the reference, using the classic method adapted from Pfurtscheller and colleagues (e.g., Pfurtscheller \& Aranibar, 1977; Pfurtscheller \& Lopes da Silva, 1999): ERD\% $=(A-R) /$ $R * 100$, where $R=$ power in the reference interval and $A=$ power in the active or task phase. Thus, desynchronisation and synchronisation were expressed as a percentage of the activity relative to the reference interval. (NB: Using this formula, ERD produces negative scores, and ERS produces positive ones.) The alpha band was split into two subbands, following the work of Klimesch and colleagues, who have consistently shown that alpha is not a homogeneous oscillation, but that different subbands are modulated by differing tasks (see Klimesch, Sauseng, \& Hanslmayr, 2007, for a review), and also in accordance with previous work on mu suppression to action observation (Cochin, Barthélémy, Roux, \& Martineau, 1999). The early and active time periods were chosen to strike a balance between exploiting the temporal resolution that EEG has to offer and limiting the number of possible statistical comparisons to be made.

Design Experiment 1 had a mixed factor design with two repeated measures factors, Stimulus Type (yawn vs. gape) and Electrode (with three levels: C2, C4, and C6), and one between-subjects factor, Empathy Group (high IRI vs. low IRI). This was also repeated for the FC-electrode strip (i.e., FC2, FC4, and FC6) for each time period (early and late) and for the CP-electrode strip (CP2, CP4, and CP6) as a control site. Where excessively noisy electrodes or those without signal were excluded from analyses, we removed all data for the affected participants from the corresponding ANOVA. Consequently, the degrees of freedom for these analyses changed to reflect the number of participants remaining. The excluded electrodes and the number of affected participants are reported in the relevant Results sections. The dependent variables were the ERD/ERS values in the low and upper alpha bandwidths. In order to explore interactions, we used one-way ANOVA planned comparisons to examine between-subjects differences, and paired Student's $t$ tests for repeated measures differences. As this first experiment was somewhat exploratory in nature, we have chosen to report findings that approached statistical significance in addition to those that were significant, in order to show as clear a picture as possible of the effects on the EEGs of people observing yawns. We also report planned post-hoc comparisons investigating interactions that approached significance, as our main interest was to see whether mu suppression to yawn observation differed according to one's level of empathy (i.e., high vs. low IRI during yawn observation, yawn vs. gape in high and low IRI). The danger of Type I error inherent in this approach is offset by using Experiments 2 and 3 to replicate these findings. 


\section{Results}

See Fig. 1 for a depiction of the results according to electrode site. For the FC-strip ANOVA, excess noise applied to FC4 in 1 participant and to FC6 in 2 participants, resulting in the exclusion of 2 participants in total (1 participant had two of the noisy electrodes). For the C-strip analysis, excess noise applied to $\mathrm{C} 6$ in 1 participant, resulting in the exclusion of that participant.

FC strip early $(0-1,000 \mathrm{~ms})$ In the low alpha bandwidth, there was a significant interaction between stimulus type and empathy group $[F(1,13)=4.84, p=.047]$. Greater ERD to yawns than to gapes was noted for the high-IRI group $[t(8)=2.36, p=.046]$, as well as greater ERD for the high-IRI than for the low-IRI group during yawns that almost reached significance $[F(1,16)=4.49, p=.051]$. No other differences were significant, nor were there any findings in the upper alpha bandwidths ( $p s>.05)$.

FC strip late (1,000-2,500 $\mathrm{ms})$ In both the low and upper alpha bandwidths, there was a significant main effect of

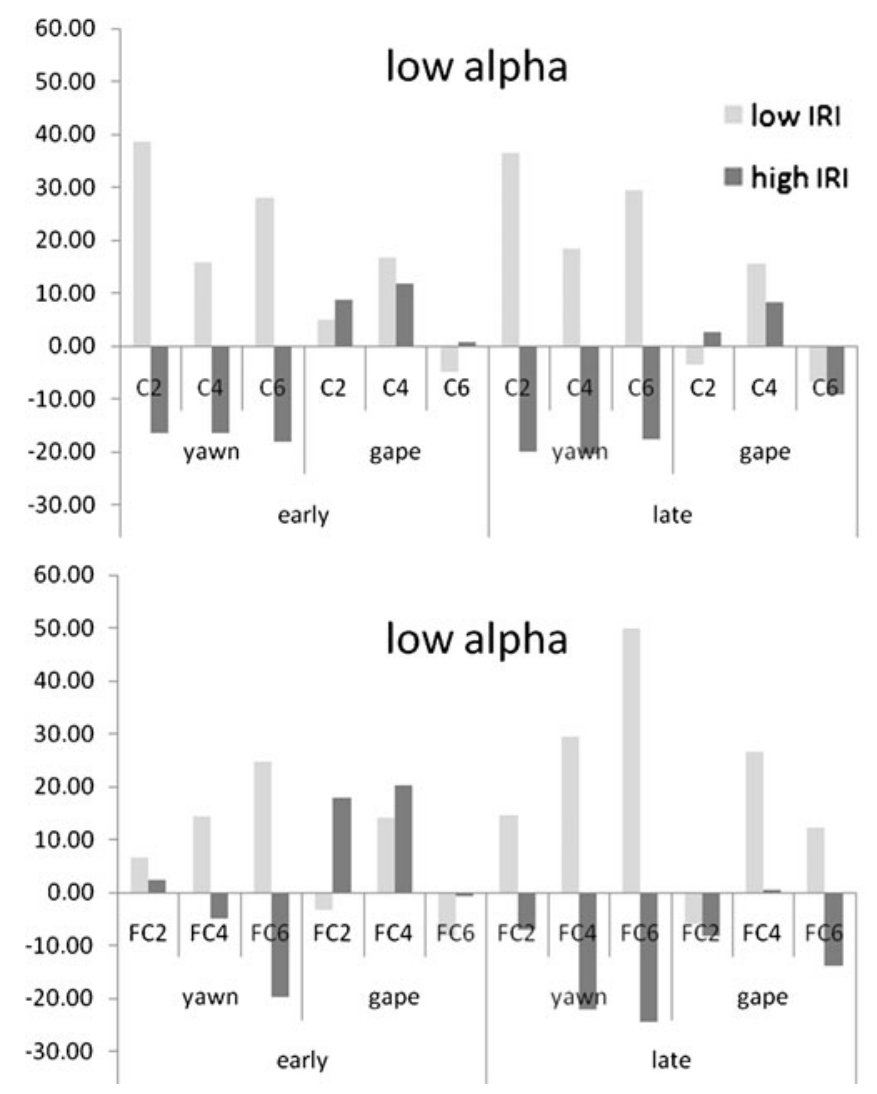

Fig. 1 Event-related desynchronisation and synchronisation (ERD/ ERS) in the low $(8-10 \mathrm{~Hz})$ and upper $(10-12 \mathrm{~Hz})$ alpha bandwidths for yawn and gape stimuli, in early and late epochs, according to electrode site and empathy group in Experiment 1. The group with high scores on the Interpersonal Reactivity Index (IRI; i.e., high empathy group, such that ERD was greater for high than for low IRI $[F(1,13)=7.57, p=.016$, and $F(1,13)=21.51$, $p<.001$, respectively], and for low alpha, this effect was driven by activation during the yawn condition $[F(1,15)=$ 4.87, $p=.043]$.

$C$ strip early (0-1,000 ms) In the low alpha bandwidth, there was a main effect of empathy group, with greater ERD for high than for low IRI $[F(1,14)=4.84, p=.045]$. This effect was modulated by an interaction with stimulus type $[F(1,14)=6.09, p=.027]$. This revealed that, similar to results for the FC strip, the empathy group effect was driven by activation during the yawn condition $[F(1,16)=$ $8.27, p=.012]$. Greater ERD in the yawn as opposed to the gape condition approached significance in the high-IRI group $[t(8)=2.14, p=.065]$.

In the upper alpha bandwidth, there was a main effect of stimulus type that approached significance, with greater ERD to yawns than to gapes $[F(1,14)=4.62, p=.050]$. This was modulated by an interaction with empathy group and electrode that also approached significance $[F(2,28)=$ $3.29, p=.052]$. In the high-IRI group, yawns elicited more
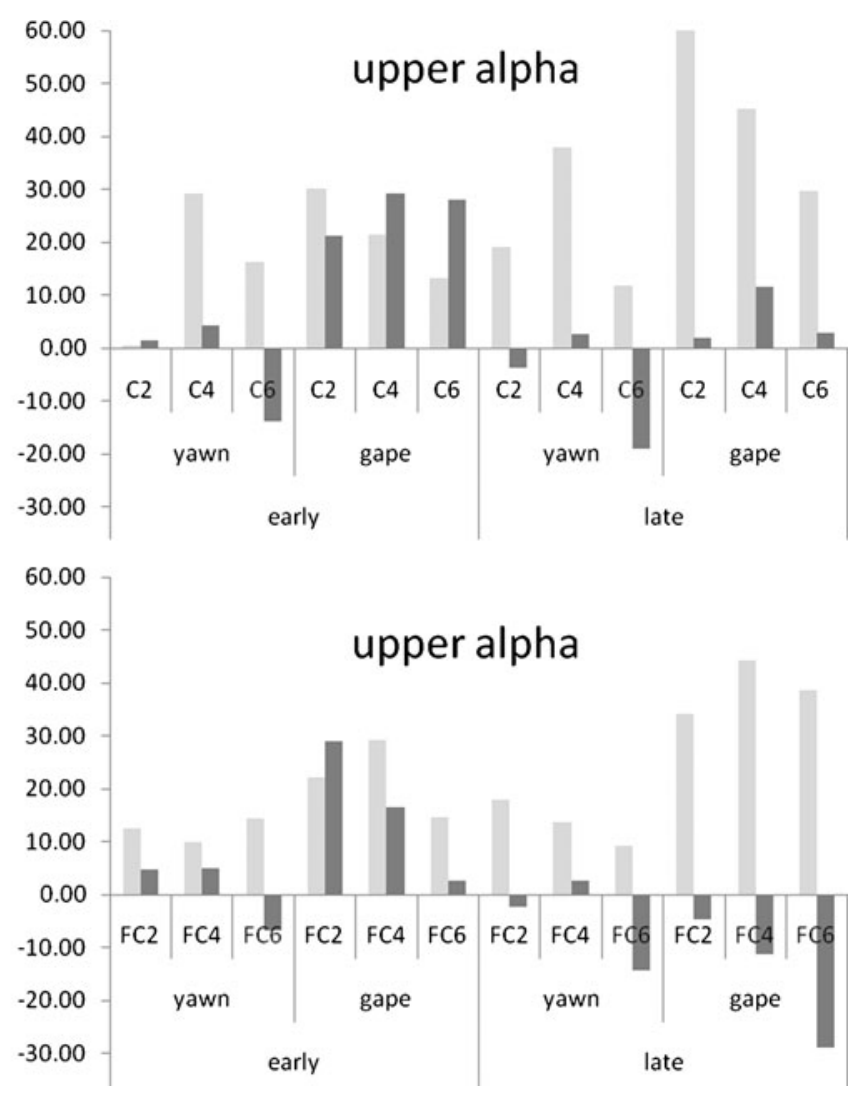

empathy) is in dark grey, and those with low IRI scores (low empathy) are in light grey. The $y$-axis represents ERD/ERS as a percentage change from the reference period (negative values $=\mathrm{ERD}$, positive values $=$ ERS) 
ERD than did gapes at electrode C6 $[t(8)=2.87, p=.021]$, and also at $\mathrm{C} 6$, during yawns, those in the high-IRI group had greater ERD than those in the low-IRI group $[F(1,15)=$ $10.99, p=.005]$.

$C$ strip late (1,000-2,500 ms) In the low alpha bandwidth, we observed that the high-IRI group exhibited greater ERD to yawns than did the low-IRI group $[F(1,15)=8.71, p=.010]$. However, the interaction between group and stimulus type did not quite reach significance $[F(1,14)=3.85, p=.07]$.

In the upper alpha bandwidth, there was a main effect of stimulus type, wherein yawns elicited greater ERD than gapes $[F(1,14)=7.97, p=.014]$, as well as a main effect of empathy group, in which greater ERD was observed in the high-IRI group. Both main effects were modulated by an interaction with each other $[F(1,14)=9.03, p=.009]$, which revealed that the group effect was driven by significantly greater ERS in the low- than in the high-IRI group during the gape condition $[F(1,16)=9.68, p=.007]$. This appears also to have been driven by significantly greater ERS in the gape as opposed to the yawn condition in the low-IRI group at electrodes $\mathrm{C} 2[t(7)=2.88, p=.024]$ and $\mathrm{C} 6[t(6)=2.94, p=.026]$.

$C P$ strip (control) In order to check that the findings in the alpha bandwidth were due to mu activity (i.e., deriving from sensorimotor areas) and not related to occipital alpha, we employed a control site over the right CP strip (CP2, CP4, and CP6). The rationale was that, if our alpha findings were the result of occipital activation, they should be stronger/ more prevalent at these electrodes. We found no significant findings at these electrodes $(p s>.05)$.

\section{Discussion}

We hypothesised that at right frontocentral sites, observation of yawn stimuli would elicit greater mu suppression (ERD) than would observing gapes (control stimuli), and that this mu suppression would be greater for those in the high-IRI group. We found evidence to support these hypotheses. At right frontal sites (the FC strip), the biggest experimental effects were observed early in the presentation of the stimulus videos $(0-1,000 \mathrm{~ms})$ in the low alpha bandwidth. Here we found greater ERD to yawns than to gapes in the highIRI group, and a close to significant finding of greater ERD for the high- than for the low-IRI group during the presentation of yawns. In the latter part of the video presentations $(1,000-2,500 \mathrm{~ms})$, we found that those in the high-IRI group exhibited more ERD than did those in the low group in both the low and upper alpha bandwidths. On closer inspection, we found that, for low alpha, this effect of group was only significant during the yawn condition, not during the gapes.
At right central sites (the $\mathrm{C}$ strip), in low alpha, we observed greater ERD in the high- than in the low-IRI group during yawn presentation during both early and late stages of video presentation. We also noted a trend for greater lowalpha ERD to yawns than to gapes in the high-IRI group in the early period. In the upper alpha bandwidth, during early video presentation, we found additional support for the hypotheses at electrode C6 (i.e., greater ERD in the highthan in the low-IRI group during yawns, and also increased ERD in yawns as opposed to gapes in the high-IRI group). However, during the late period of video presentation, the results are somewhat more ambiguous: We did observe a significant difference between yawns and gapes in the proposed direction, but this seems to have been driven, at least in part, by an increase in mu power (ERS) in the low-IRI group during the presentation of the gape videos. Indeed, in this condition, there was also a significant difference between the empathy groups (greater ERS for the low- than for the high-IRI group).

To summarise, the low-alpha results from right frontocentral electrodes were in accordance with our hypotheses at both early and late time points. The upper-alpha results support the hypotheses from right frontal sites during the latter part of the video presentations and from right lateral central sites early in the presentations. However, right central sites show a different pattern of activation during the latter section of the stimulus presentation, with those in the low-IRI group exhibiting an increase in upper-alpha power (ERS). The fact that no significant differences in the bandwidths of interest were noted in the more posterior CP strip indicates that we can be reasonably assured that the patterns of activation that were observed in the $\mathrm{C}$ and $\mathrm{FC}$ electrodes resulted from changes in mu activation and not from more caudal occipital alpha.

\section{Experiment 2}

In this experiment, we sought to investigate the hypothesis of an involvement of the human mirror neuron system (hMNS) during contagious yawning in the general population. Towards this aim, we recruited a larger study population and did not select according to extremes in empathy levels. Again, we used EEG indices of hMNS activation (mu suppression) as our main dependent variables, hypothesising greater mu suppression to yawns than to control stimuli (gapes).

\section{Method}

Participants A group of 36 participants (16 female) took part in the study, with an age range of 18-44 years (mean = 24.9). Participants gave written informed consent. The study was approved by the University of Essex Ethics Committee. 
Materials and procedure All of the materials and procedures were identical to those of Experiment 1, except that the IRI was not employed.

EEG acquisition and preparation The EEG acquisition and preparation procedures were the same as for Experiment 1, except that only 14 scalp sites were recorded from (FC5, FC3, $\mathrm{FC} 1, \mathrm{FCz}, \mathrm{FC} 2, \mathrm{FC} 4, \mathrm{FC} 6, \mathrm{C} 5, \mathrm{C} 3, \mathrm{C} 1, \mathrm{Cz}, \mathrm{C} 2, \mathrm{C} 4$, and C6).

\section{Results}

See Fig. 2 for a depiction of the results according to electrode site. For the FC-strip ANOVA, excess noise applied to FC2 in 1 participant, to FC4 in 1 participant, and to FC6 in 6 participants, resulting in the exclusion of 7 participants in total (1 participant had two noisy electrodes). For the C-strip analysis, excess noise applied to $\mathrm{C} 2$ in 2 participants and to C6 in 6 participants, resulting in the exclusion of 7 participants in total (1 participant had two noisy electrodes).

FC strip early (0-1,000 ms) There were no significant differences of interest in the low or upper alpha bandwidths $(p \mathrm{~s}>.05)$.

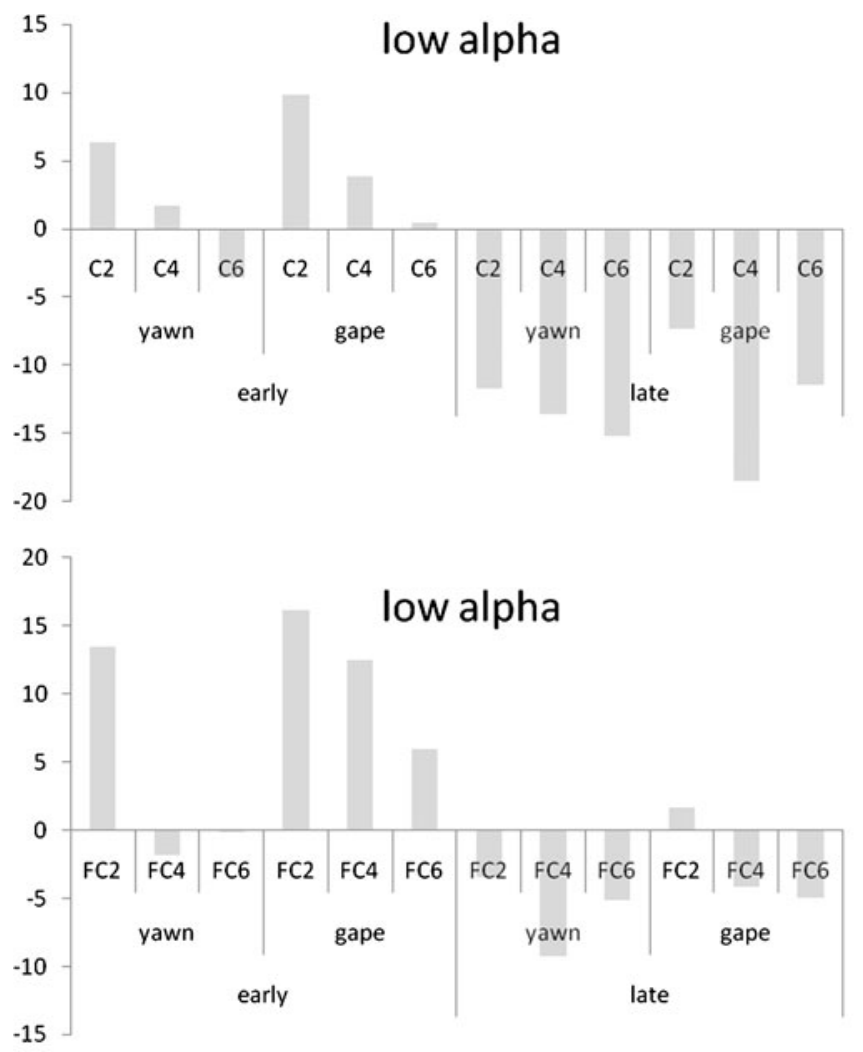

Fig. 2 Event-related desynchronisation and synchronisation (ERD/ ERS) in the low $(8-10 \mathrm{~Hz})$ and upper $(10-12 \mathrm{~Hz})$ alpha bandwidths for yawn and gape stimuli, in early and late epochs, according to
FC strip late (1,000-2,500 $\mathrm{ms})$ In the upper alpha bandwidth, an interaction between stimulus type and electrode $[F(2,56)=4.98, p=.01]$ was noted, but further investigation revealed no significant differences of interest ( $p$ s $>.05)$.

C strip early $(0-1,000 \mathrm{~ms})$ There were no significant differences of interest in any bandwidth $(p s>.05)$.

$C$ strip late (1,000-2,500 ms) There was a main effect of stimulus type in the upper alpha bandwidth, revealing greater ERD to yawns than to gapes $[F(1,28)=5.29, p=.029]$.

\section{Discussion}

In Experiment 2, we observed greater upper-alpha ERD to yawns than to control stimuli over more central areas during the latter part of the video presentations. This finding replicates that found in Experiment 1, and is in agreement with our hypotheses and with predictions of hMNS involvement in contagious yawning in the general population. It should be noted, however, that the pattern of results from this experiment was narrower than that from Experiment 1, and
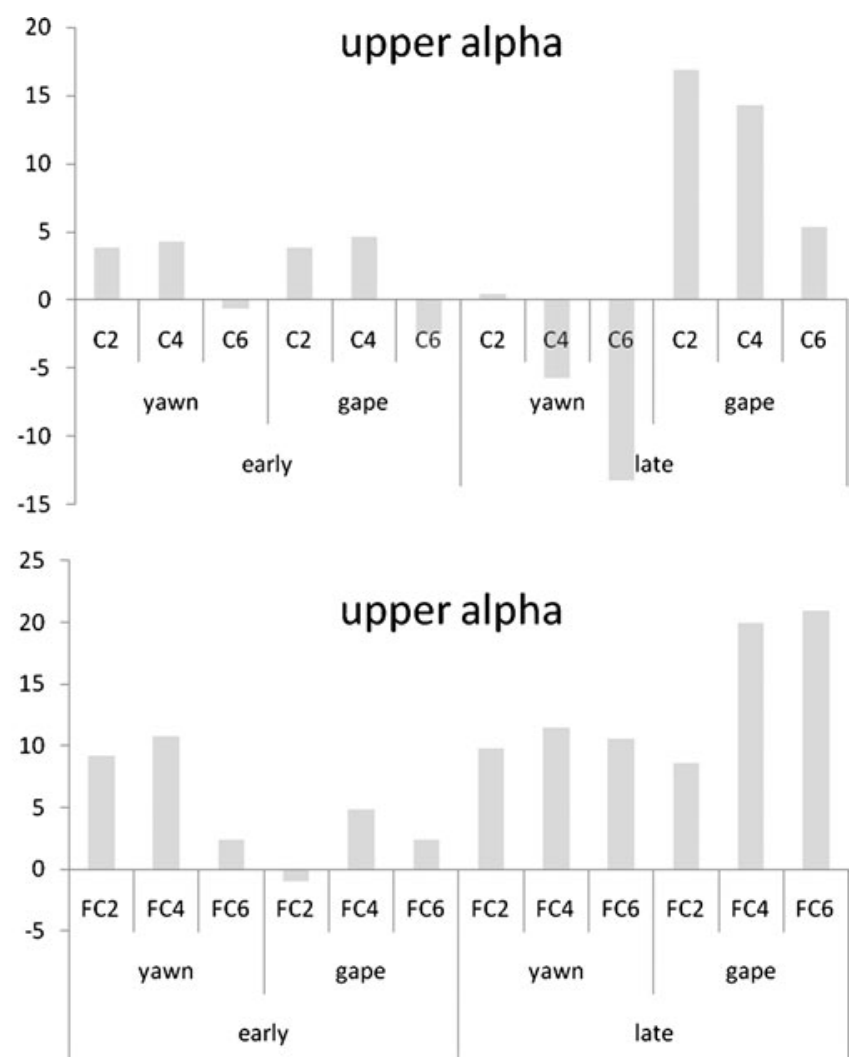

electrode site in Experiment 2. The $y$-axis represents ERD/ERS as a percentage change from the reference period (negative values $=E R D$, positive values $=$ ERS) 
many fewer significant effects were observed. This is not surprising, because in the first experiment, the results were divided and analysed according to empathy group, and therefore were more sensitive to individual differences in hMNS activation. Nevertheless, in both experiments, main effects of stimulus type were observed, notably upper alpha ERD over the $\mathrm{C}$ strip in the latter part of the video presentations.

In summary, using EEG methods, we have found evidence that is consistent with a possible role for hMNS activation in the general population. This is in agreement with Arnott et al. (2009), but not with other fMRI studies that have argued against hMNS involvement in contagious yawning (Nahab et al., 2009; Platek et al., 2005; Schürmann et al., 2005) or that showed no further increase in hMNS activation in comparison to control conditions (Nahab et al., 2009; Schürmann et al., 2005). Arnott et al. previously proposed that one possible reason why previous investigations have failed to observe the specific involvement of hMNS during yawn observation was their choice of control stimuli that would also activate the hMNS. This may well be a factor in imaging studies, but given our results from the first two experiments, it does not seem to be the case for EEG investigations. It is possible that the observation of yawns induces changes in the EEG mu rhythm with a greater signal-to-noise ratio than is present in the induced changes to the BOLD signal measured by fMRI. Alternatively, changes in neural synchrony (and, hence, in the EEG signal) can occur without large changes in cortical energy consumption (Hari, 1996; Hari et al., 1998), so the differences in findings between the present study and the three previous fMRI studies cited above may simply result from the fact that EEG and fMRI can measure different expressions of activation. In a third experiment, we set out to investigate whether EEG was also a suitable tool to examine the relationship between contagious yawning and the hMNS when using auditory stimuli similar to those employed by Arnott et al. (2009).

\section{Experiment 3}

Arnott and colleagues (2009) proposed that the discrepancies between studies with regard to hMNS involvement in contagious yawning might be due in part to the choice of control stimuli (see above). However, in our first two experiments, we found that evidence consistent with hMNS activation during the observation of yawns can be observed when using EEG methodologies. In a third experiment, we set out to examine hMNS reactivity using a version of the Arnott auditory protocol adapted for EEG. If the earlier fMRI findings were influenced by the control stimuli, we should expect stronger findings than in our previous two experiments.
Given the previous findings of Senju et al. (2007) of decreased susceptibility to contagious yawning in children with ASD, we also chose to investigate the influence of autistic traits in the general population on mu suppression during the observation of yawns. To this end, we employed the Autism Spectrum Quotient (AQ; Baron-Cohen, Wheelwright, Skinner, Martin, \& Clubley, 2001), and participants were also given the Interpersonal Reactivity Index (IRI; Davis, 1983) as a measure of empathy. In light of Kaplan and Iacoboni's (2006) observations of right frontal hMNS activation correlated with the Empathic Concern (EC) subscale of the IRI, we focused our attention on this subscale. We expected to find increased mu suppression to yawns for those scoring highly on EC (i.e., greater levels of empathy) and those with low AQ scores (i.e., fewer autistic traits).

\section{Method}

Participants A group of 20 participants (10 female) took part in the study, with a mean age 23 years $(S D=5)$. The participants gave written informed consent. During the data analysis, 1 participant was excluded for excessively noisy data, resulting in the data from 19 participants being analysed. The study was approved by the University of Essex Ethics Committee.

Materials Empathy levels were measured using the IRI (Davis, 1983). Levels of autistic traits were measured using the AQ (Baron-Cohen et al., 2001). The experimental stimuli were either 3-s-long auditory recordings of people yawning or electronically scrambled versions of the yawns that served as control stimuli (see Arnott et al., 2009). We used a total of 14 different stimuli (7 yawns and 7 scrambled stimuli). Each auditory clip was presented four times in a randomised order, amounting to 56 trials in total. Each trial consisted of the presentation of (1) a fixation cross $(2,000 \mathrm{~ms} ; 25 \times 20 \mathrm{~mm})$, (2) the auditory clip $(3,000 \mathrm{~ms})$, and (3) a blank screen $(3,000 \mathrm{~ms})$; thus, each trial lasted $8 \mathrm{~s}$. The stimulus presentation software and hardware were the same that were used in Experiments 1 and 2.

Procedure We seated participants in a darkened, quiet room, $70 \mathrm{~cm}$ in front of the computer monitor. EEG was recorded while they first completed an eye movement calibration protocol (Croft \& Barry, 2000), followed by 2 min rest with eyes closed, followed by the auditory yawn protocol. In order to ensure that attention was sustained whilst listening to the recordings and that eyes were kept open throughout the experiment, participants were instructed to count silently to themselves the number of times that they saw the fixation cross appear. There were eight additional "mock" trials on which only the fixation cross appeared and no subsequent 
stimuli were presented. These were randomly presented throughout the experiment. Thus, participants needed to keep their eyes open and to stay alert in order to carry out the task accurately.

EEG data acquisition EEG data were recorded with Neuroscan 4.4 acquisition software and SynampsII amplifiers, from 17 scalp sites (FC5, FC3, FC1, FCz, FC2, FC4, FC6, $\mathrm{C} 5, \mathrm{C} 3, \mathrm{C} 1, \mathrm{Cz}, \mathrm{C} 2, \mathrm{C} 4, \mathrm{C} 6, \mathrm{M} 1, \mathrm{M} 2$, and $\mathrm{Oz}$ ) using a Quick-Cap arranged according to the international 10-10 system (Compumedics, Melbourne, Australia). All other parameters were as in Experiment 1.

EEG data preparation All data preparation was as for Experiment 1, except that (1) data were prepared using Neuroscan Edit 4.4 (Compumedics, Melbourne, Australia) and (2) the data were epoched from $-2,500$ to $4,000 \mathrm{~ms}$ around the start of each video clip, and a reference interval of $-1,500$ to $-477 \mathrm{~ms}$ was used to calculate ERD/ERS.

Design Experiment 3 had a repeated measures design with two factors: Stimulus Type (yawn vs. gape) and Electrode (with three levels: C2, C4, and C6). This was also repeated

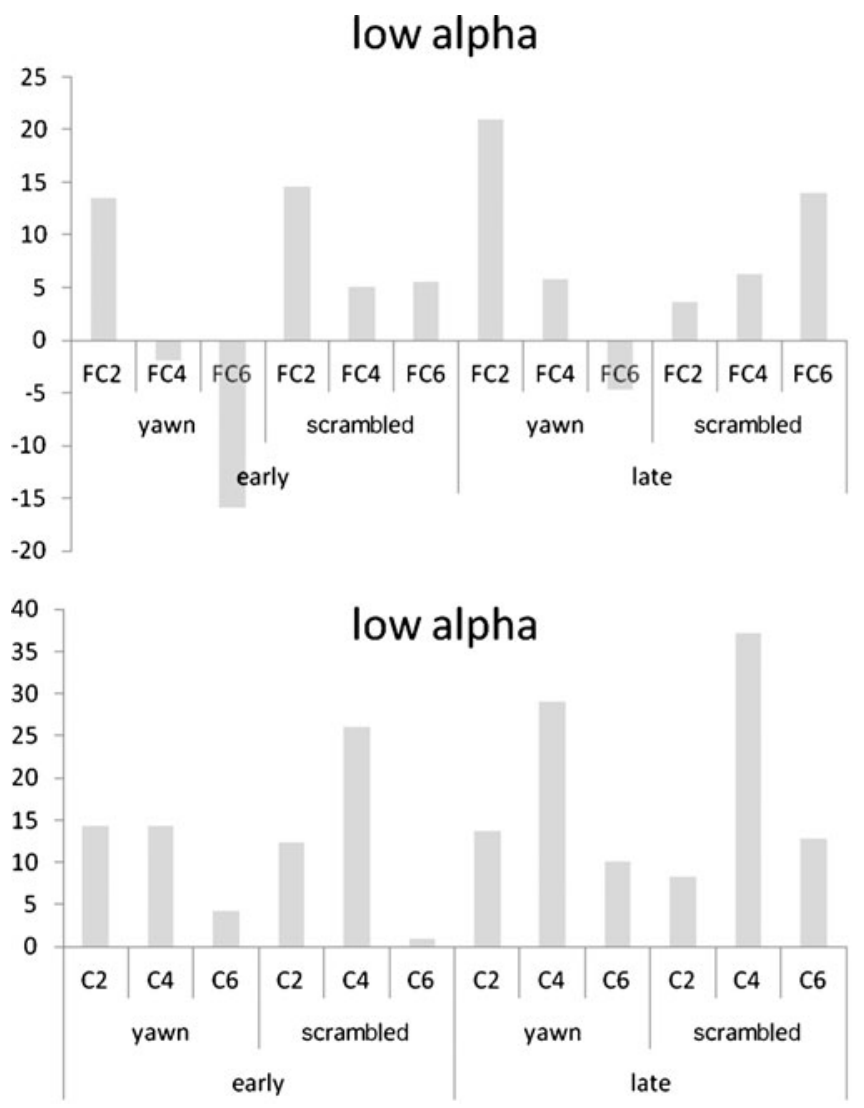

Fig. 3 Event-related desynchronisation and synchronisation (ERD/ ERS) in the low $(8-10 \mathrm{~Hz})$ and upper $(10-12 \mathrm{~Hz})$ alpha bandwidths for auditory yawn and scrambled stimuli, in early and late epochs, for the FC electrode strip (i.e., FC2, FC4, and FC6), for each time period (early and late), and for the $\mathrm{Oz}$ electrode as a control site. A secondary analysis was also performed using a median split on scores on the EC subscale of the IRI, resulting in a between-subjects factor, EC. The dependent variables and planned comparisons were as in the previous two experiments.

\section{Results}

See Fig. 3 for a depiction of results according to electrode site. For the FC-strip ANOVA, excess noise applied to FC6 in 4 participants, resulting in the exclusion of those participants.

FC strip early $(0-1,000 \mathrm{~ms})$ There were no significant differences of interest in any bandwidth $(p s>.05)$.

FC strip late (1,000-2,500 ms) In the low alpha band, there was a significant interaction between stimulus type and electrode $[F(2,28)=3.71, p=.037]$. The only significant difference was between the yawns and scrambled stimuli at electrode FC6 $[t(14)=2.15, p=.05]$. We also investigated

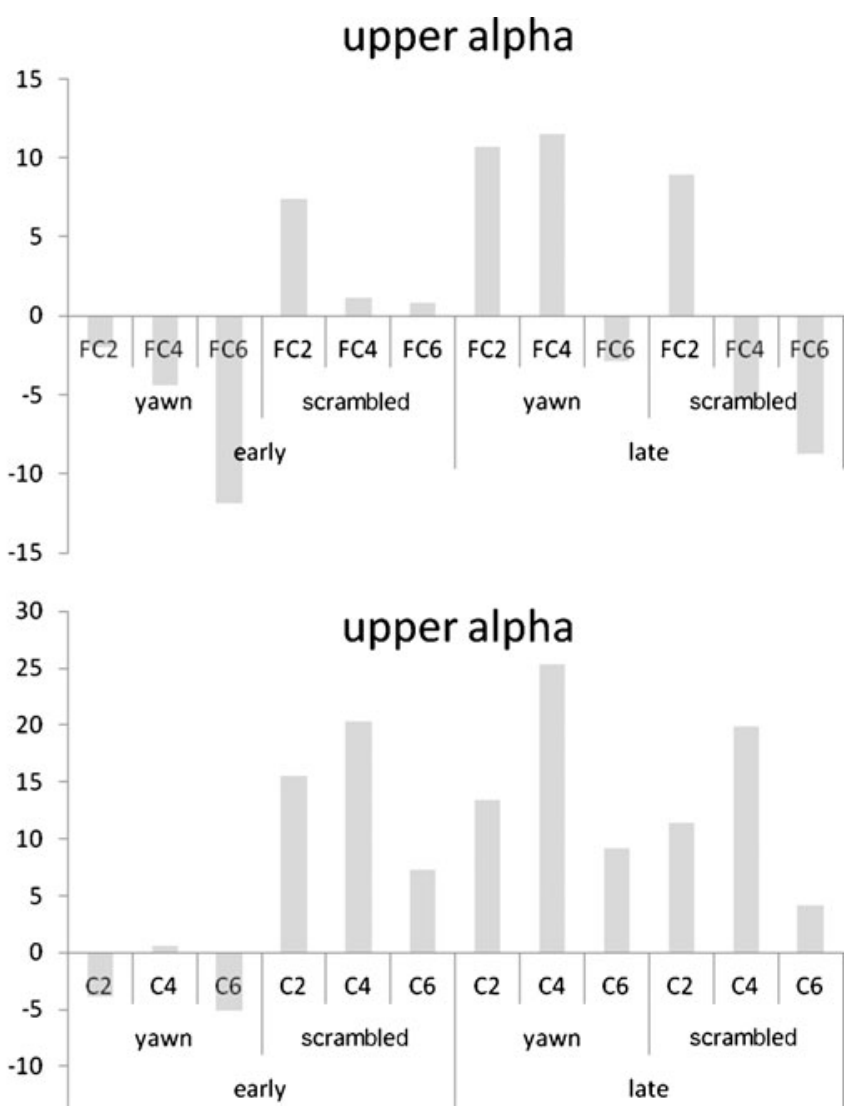

according to electrode site in Experiment 3. The $y$-axis represents $\mathrm{ERD} / \mathrm{ERS}$ as a percentage change from the reference period (negative values $=$ ERD, positive values $=$ ERS) 
the effect of EC on mu suppression. EC significantly interacted with stimulus type $[F(1,12)=5.25, p=.041]$ and was driven by significantly greater low-alpha ERD to yawns than to scrambled stimuli for those scoring highly on empathic concern $[t(9)=2.39, p=.04]$.

Also for low alpha, scores on the AQ correlated with ERD in the right FC strip, such that the lower the AQ score, the greater the $\operatorname{ERD}(r=.610, n=18 ; p=.007)$.

$C$ strip early (0-1,000 ms) There were no significant differences of interest in any bandwidth $(p \mathrm{~s}>.05)$.

$C$ strip late $(1,000-2,500 \mathrm{~ms})$ There were no significant differences of interest in any bandwidth $(p s>.05)$.

\section{Discussion}

In Experiment 3, we observed greater low-alpha ERD to yawns than to control stimuli over right frontal areas during the latter part of the video presentations. These results are in line with recent fMRI findings of increased right inferior frontal gyrus activation (part of the hMNS) during exposure to yawn stimuli (Arnott et al., 2009). Our results appear to have been driven to some extent by the mu reactivity of those scoring highly for EC. This is pertinent, given Kaplan and Iacoboni's (2006) findings of a correlation between EC and activation in inferior right frontal areas of the hMNS. It was also interesting to note a negative correlation between $\mathrm{AQ}$ and low-alpha ERD. Thus, having more autistic traits was related to exhibiting less mu suppression when observing yawns. In light of our hypothesis, this fits well with previous findings of reduced contagious yawning in children with ASD (Senju et al., 2007). Thus, the parsimonious interpretation of our results is that, again, they partially support a role for hMNS in contagious yawning. Indeed, the notion of a developing hMNS playing a role in contagious yawning (or no role, as in ASD) may also shed some light on the observation that children tend not to succumb to contagious yawning until around the age of 4-5 years (Anderson \& Meno, 2003; Helt, Eigsti, Snyder, \& Fein, 2010). Many have argued that the hMNS develops through sensorimotor learning and classic Hebbian principles (Catmur, Walsh, \& Heyes, 2007; Gillmeister, Catmur, Liepelt, Brass, \& Heyes, 2008; Heyes, 2010) from early simple mimicry to later "functional context-sensitivities during action observation" (Pineda, 2008, p. 13). Thus, we would not expect a fully functioning contagious yawning response in early childhood but would expect this response to mature with the development of the hMNS with learning. Indeed, a learned associative hMNS response to observing yawns would also fit well with various proposed adaptive benefits of contagious yawning (e.g., to synchronise group arousal/vigilance levels).
With regard to the more technical issues of EEG/fMRI sensitivity and suitable control stimuli, the fact that our results are in line with those of Arnott et al. (2009) would seem, on face value, to support the notion that there was a problem with the control stimuli in previous studies. However, the findings from Experiment 3 are not stronger than those from Experiments 1 and 2, so this simple interpretation is unlikely. Our results suggest that a combination of factors may be at play. Firstly, EEG may be picking up different activation from that measured by fMRI. Secondly, the results from Experiment 1 were stronger than those from Experiments 2 and 3. This would appear to result from the fact that participants were grouped according to their empathy scores, in terms of one standard deviation above or below the mean. It is clear that empathy affects the degree to which the hMNS is activated, and by dividing the participants in this way, we were better able to account for individual differences in mirror neuron activation. Using a median split, as in Experiment 3, or making no differentiation for empathy, as in Experiment 2, was less effective. Previous fMRI studies that have not accounted for individual differences in this way may have been less likely to observe experimental effects. Thirdly, another possible reason for the weaker results in Experiment 3 is that there still could be a problem with the control stimuli. Despite the scrambled auditory stimuli being described as "choppy, unnatural and not contagious" (Arnott et al., 2009, p. 336), there was still some humanity to the vocalisations, and participants might have attempted to try to make some sense of them, potentially resulting in activated mirror neuron networks. Additionally, since the scrambled stimuli were "choppy," they might have contained transients that added noise to the signal. Arguably, then, the ideal control for yawning stimuli has still not been realised and remains a high priority for future work in this area.

\section{General discussion}

In three experiments, in line with our predictions, we demonstrated greater mu suppression over right frontocentral areas when participants were exposed to yawns as opposed to control stimuli. We also noted that those who score highly on measures of empathy tend to exhibit greater suppression of their ongoing mu activity than do those with low scores, and that this appears to be particularly evident during yawn stimuli. Similarly, we observed with an increase in autistic traits, a corresponding decrease in mu suppression (less desynchronisation), and we suggest that this effect may underlie the decrease in contagious yawning noted in ASD (Senju et al., 2007). In the context of action observation, mu suppression is regarded as a reliable index of mirror neuron activation (Kilner et al., 2009; Muthukumaraswamy \& Johnson, 2004; Pineda, 2005, 2008). Consequently, the parsimonious interpretation of our data is that the human mirror neuron system (hMNS) is 
activated when observing yawns, and we suggest that this system may underlie the contagious aspects of the phenomenon. This interpretation is in accordance with Arnott et al. (2009), who found increased BOLD activation to the sound of yawns in right inferior frontal gyrus (a core component of the hMNS), but not with three other neuroimaging studies, which found no evidence for hMNS activation during contagious yawning above that found during exposure to control stimuli (Nahab et al., 2009; Platek et al., 2005; Schürmann et al., 2005). We propose three possible reasons why those studies might have failed to find hMNS involvement in contagious yawning: Firstly, EEG provides a different method for investigating cortical activation to yawn stimuli (i.e., neural synchrony, as opposed to changes in blood oxygen levels); secondly, the control stimuli used were also likely to activate hMNS, and therefore might have obscured the results; thirdly, individual differences in personality traits such as levels of empathy were not built in to the studies' factorial designs.

In the present study, the findings of greater mu suppression during exposure to yawn stimuli for those who score highly on measures of empathy fit well with the previous literature linking contagious yawning with empathy (Platek et al., 2003; Senju et al., 2007), and also with studies correlating empathy with hMNS activation (e.g., Gazzola et al., 2006; Kaplan \& Iacoboni, 2006; Pfeifer et al., 2008). This is particularly so for the low alpha band over right frontal areas during the later part of the stimulus presentation, where this effect was found first in Experiment 1 and was replicated in Experiment 3. Thus, both experiments that examined empathy observed this effect. Despite the support for our hypotheses that our data provide, some limitations do need to be acknowledged. For instance, not all of the findings from Experiment 1 were replicated in the later experiments. For example, the near significant $(p=.05)$ finding of greater upper-alpha ERD to yawns than to controls over right central electrodes during the early part of the video presentation was not found again. However, a similar effect in the later part of the video presentation was replicated in Experiment 2. Clearly, other significant effects found in Experiment 1 pertaining to individual levels in empathy would not be expected to be observed in Experiment 2 (where empathy was not measured). Additionally, in Experiment 3, a median split for empathic concern was used to divide the data, and so would have had less power than creating groups based on separations of one standard deviation from the mean (as in Exp. 1), and therefore the failure to replicate findings may be attributable to this. Furthermore, differences in the findings between the three experiments may also have resulted from differences in experimental modality (e.g., visual vs. auditory stimuli). We are also aware that the control stimuli we used were still not optimal and might have also activated hMNS (albeit to a lesser extent than the yawn stimuli); this might have diluted our findings. The creation of a definitive control condition for yawns remains a high priority for researchers in this field. It is also important to consider the possibility that the observed mu suppression might have been caused by mechanisms other than hMNS. For instance, if networks involved in other social cognition skills (e.g., theory of mind) created a motor command to yawn in response to observing a yawn, this, too, would excite the motor cortex, leading to a desynchronisation of mu activity. Indeed, given that various theory-of-mind behaviours (especially with regard to affect) have recently been associated with activation of the ventromedial prefrontal cortex (vmPFC; Abu-Akel \& Shamay-Tsoory, 2011; Lev-Ran, ShamayTsoory, Zangen, \& Levkovitz, in press), such an explanation could bind our results in the present study with those of Nahab et al. (2009), who found vmPFC activation to yawn observation. Additionally, it has recently been argued that hMNS and non-mirror theory-of-mind networks work together in a complementary fashion to facilitate the understanding of actions (de Lange, Spronk, Willems, Toni, \& Bekkering, 2008; Schippers, Roebroeck, Renken, Nanetti, \& Keysers, 2010); future work on contagious yawning should explore this possibility. However, to date, and to the best of our knowledge, no such link between vmPFC and motor cortex activation has been reported in this context, and the most widely published explanation of mu suppression to observation of an action is downstream modulation of motor cortex by premotor mirror neurons (Pineda, 2008; Rizzolatti \& Craighero, 2004), although recently, direct mirror-neuron-like activity has been observed in M1 itself (Dushanova \& Donoghue, 2010; Press et al., 2011). Therefore, at present, the most plausible explanation of our data is in terms of hMNS activation during the observation of yawns, but this should not preclude the investigation of a possible link between vmPFC and hMNS in future studies.

In summary, we have presented evidence of greater mu suppression to observing yawn stimuli than to observing control stimuli. Given an interpretation of the desynchronisation of mu power as a putative index of hMNS activation, our results are consistent with previous findings by Arnott et al. (2009) implicating the human mirror neuron system in the phenomenon of contagious yawning. This is particularly apparent when controlling for individual differences in empathic abilities. Future studies in this field will need to take these findings into account and also to design control stimuli that do not activate the hMNS.

Author note We thank Roger Grace, Roger Deeble, and Mike Lodge for their excellent technical support for these studies.

\section{References}

Abu-Akel, A., \& Shamay-Tsoory, S. (2011). Neuroanatomical and neurochemical bases of theory of mind. Neuropsychologia, 49, 2971-2984. 
Anderson, J. R., \& Meno, P. (2003). Psychological influences on yawning in children. Current Psychology Letters, 11(2).

Arnott, S. R., Singhal, A., \& Goodale, M. A. (2009). An investigation of auditory contagious yawning. Cognitive, Affective, \& Behavioral Neuroscience, 9, 335-342.

Arroyo, S., Lesser, R. P., Gordon, B., Uematsu, S., Jackson, D., \& Webber, R. (1993). Functional significance of the mu rhythm of human cortex: An electrophysiologic study with subdural electrodes. Electroencephalography and Clinical Neurophysiology, 87, 76-87.

Baron-Cohen, S., Wheelwright, S., Skinner, R., Martin, J., \& Clubley, E. (2001). The autism-spectrum quotient (AQ): Evidence from Asperger syndrome/high-functioning autism, males and females, scientists and mathematicians. Journal of Autism and Developmental Disorders, 31, 5-17.

Catmur, C., Walsh, V., \& Heyes, C. (2007). Sensorimotor learning configures the human mirror system. Current Biology, 17, 1527-1531.

Chatrian, G. E., Petersen, M. C., \& Lazarte, J. A. (1959). The blocking of the rolandic wicket rhythm and some central changes related to movement. Electroencephalography and Clinical Neurophysiology, 11, 497-510.

Cochin, S., Barthélémy, C., Roux, S., \& Martineau, J. (1999). Observation and execution of movement: Similarities demonstrated by quantified electroencephalography. European Journal of Neuroscience, 11, 1839-1842.

Cooper, N. R., Puzzo, I., \& Pawley, A. D. (2008). Contagious yawning: The mirror neuron system may be a candidate physiological mechanism. Medical Hypotheses, 71, 975-976.

Croft, R. J., \& Barry, R. J. (2000). EOG correction of blinks with saccade coefficients: A test and revision of the aligned-artefact average solution. Clinical Neurophysiology, 111, 444-451.

Davis, M. H. (1983). Measuring individual differences in empathy: Evidence for a multidimensional approach. Journal of Personality and Social Psychology, 44, 113-126.

de Lange, F. P., Spronk, M., Willems, R. M., Toni, I., \& Bekkering, H. (2008). Complementary systems for understanding action intentions. Current Biology, 18, 454-457.

di Pellegrino, G., Fadiga, L., Fogassi, L., Gallese, V., \& Rizzolatti, G. (1992). Understanding motor events: A neurophysiological study. Experimental Brain Research, 91, 176-180.

Dushanova, J., \& Donoghue, J. (2010). Neurons in primary motor cortex engaged during action observation. European Journal of Neuroscience, 31, 386-398.

Enticott, P. G., Hoy, K. E., Herring, S. E., Johnston, P. J., Daskalakis, Z. J., \& Fitzgerald, P. B. (2008). Reduced motor facilitation during action observation in schizophrenia: A mirror neuron deficit? Schizophrenia Research, 102, 116-121.

Gallese, V., Fadiga, L., Fogassi, L., \& Rizzolatti, G. (1996). Action recognition in the premotor cortex. Brain, 119(Pt. 2), 593-609.

Gastaut, H. (1952). Étude electrocorticographique de la réactivité des rythmes rolandiques. Revue Neurologique, 87, 176-182.

Gastaut, H. J., \& Bert, J. (1954). EEG changes during cinematographic presentation. Electroencephalography and Clinical Neurophysiology, 6, 433-444.

Gazzola, V., Aziz-Zadeh, L., \& Keysers, C. (2006). Empathy and the somatotopic auditory mirror system in humans. Current Biology, 16, 1824-1829.

Gillmeister, H., Catmur, C., Liepelt, R., Brass, M., \& Heyes, C. (2008). Experience-based priming of body parts: A study of action imitation. Brain Research, 1217, 157-170.

Haker, H., \& Rossler, W. (2009). Empathy in schizophrenia: Impaired resonance. European Archives of Psychiatry and Clinical Neurosciences, 259, 352-361.

Hari, R. (1996). In I. Hashimoto, Y. Okada, \& G. Ogawa (Eds.), Visualization of information processing in the human brain: Recent advances in MEG and functional MRI (pp. 47-54). New York: Elsevier.
Hari, R., Forss, N., Avikainen, S., Kirveskari, E., Salenius, S., \& Rizzolatti, G. (1998). Activation of human primary motor cortex during action observation: A neuromagnetic study. Proceedings of the National Academy of Sciences, 95, 15061-15065.

Helt, M. S., Eigsti, I. M., Snyder, P. J., \& Fein, D. A. (2010). Contagious yawning in autistic and typical development. Child Development, 81, 1620-1631.

Heyes, C. (2010). Where do mirror neurons come from? Neuroscience and Biobehavioral Reviews, 34, 575-583.

Jasper, H., \& Penfield, W. (1949). Electrocorticograms in man: Effect of voluntary movement upon the electrical activity of the precentral gyrus. Archiv für Psychiatrie und Zeitschrift Neurologie, 183, 163-174. doi:10.1007/BF01062488

Jellema, T., Baker, C. L., Oram, M. W., \& Perrett, D. I. (2002). Cell populations in the banks of the superior temporal sulcus of the macaque monkey and imitation. In A. N. Melzoff \& W. Prinz (Eds.), The imitative mind: Development, evolution and brain bases. Cambridge: Cambridge University Press.

Kaplan, J. T., \& Iacoboni, M. (2006). Getting a grip on other minds: Mirror neurons, intention understanding, and cognitive empathy. Social Neuroscience, 1, 175-183.

Kilner, J. M., Marchant, J. L., \& Frith, C. D. (2009). Relationship between activity in human primary motor cortex during action observation and the mirror neuron system. PLoS One, 4, e4925.

Klimesch, W., Sauseng, P., \& Hanslmayr, S. (2007). EEG alpha oscillations: The inhibition-timing hypothesis. Brain Research Reviews, $53,63-88$.

Lev-Ran, S., Shamay-Tsoory, S. G., Zangen, A., \& Levkovitz, Y. (in press). Transcranial magnetic stimulation of the ventromedial prefrontal cortex impairs theory of mind learning. European Psychiatry. doi:10.1016/j.eurpsy.2010.11.008

Martineau, J., Andersson, F., Barthélémy, C., Cottier, J.-P., \& Destrieux, C. (2010). Atypical activation of the mirror neuron system during perception of hand motion in autism. Brain Research, 1320, 168175. doi:10.1016/j.brainres.2010.01.035

Mukamel, R., Ekstrom, A. D., Kaplan, J., Iacoboni, M., \& Fried, I. (2010). Single-neuron responses in humans during execution and observation of actions. Current Biology, 20, 750-756.

Muthukumaraswamy, S. D., \& Johnson, B. W. (2004). Primary motor cortex activation during action observation revealed by wavelet analysis of the EEG. Clinical Neurophysiology, 115, $1760-1766$.

Nahab, F. B. (2010). Exploring yawning with neuroimaging. Frontiers of Neurology and Neuroscience, 28, 128-133.

Nahab, F. B., Hattori, N., Saad, Z. S., \& Hallett, M. (2009). Contagious yawning and the frontal lobe: An fMRI study. Human Brain Mapping, 30, 1744-1751.

Oberman, L. M., Hubbard, E. M., McCleery, J. P., Altschuler, E. L., Ramachandran, V. S., \& Pineda, J. A. (2005). EEG evidence for mirror neuron dysfunction in autism spectrum disorders. Cognitive Brain Research, 24, 190-198.

Perry, A., \& Bentin, S. (2009). Mirror activity in the human brain while observing hand movements: A comparison between EEG desynchronization in the mu-range and previous fMRI results. Brain Research, 1282, 126-132.

Pfeifer, J. H., Iacoboni, M., Mazziotta, J. C., \& Dapretto, M. (2008). Mirroring others' emotions relates to empathy and interpersonal competence in children. NeuroImage, 39, 2076-2085. doi:10.1016/ j.neuroimage.2007.10.032

Pfurtscheller, G., \& Aranibar, A. (1977). Event-related cortical desynchronization detected by power measurements of scalp EEG. Electroencephalography and Clinical Neurophysiology, 42, 817-826.

Pfurtscheller, G., \& Lopes da Silva, F. H. (1999). Event-related EEG/ MEG synchronization and desynchronization: Basic principles. Clinical Neurophysiology, 110, 1842-1857. 
Pfurtscheller, G., Neuper, C., Brunner, C., \& da Silva, F. L. (2005). Beta rebound after different types of motor imagery in man. Neuroscience Letters, 378, 156-159.

Pineda, J. A. (2005). The functional significance of mu rhythms: Translating "seeing" and "hearing" into "doing". Brain Research Reviews, 50, 57-68.

Pineda, J. A. (2008). Sensorimotor cortex as a critical component of an "extended" mirror neuron system: Does it solve the development, correspondence, and control problems in mirroring? Behavioral and Brain Functions, 4(47), 1-16. doi:10.1186/1744-9081-4-47

Platek, S. M. (2010). Yawn, yawn, yawn, yawn; yawn, yawn, yawn! The social, evolutionary and neuroscientific facets of contagious yawning. Frontiers of Neurology and Neuroscience, 28, 107-112.

Platek, S. M., Critton, S. R., Myers, T. E., \& Gallup, G. G. (2003). Contagious yawning: The role of self-awareness and mental state attribution. Cognitive Brain Research, 17, 223-227.

Platek, S. M., Mohamed, F. B., \& Gallup, G. G., Jr. (2005). Contagious yawning and the brain. Cognitive Brain Research, 23, 448-452.

Press, C., Cook, J., Blakemore, S. J., \& Kilner, J. (2011). Dynamic modulation of human motor activity when observing actions. Journal of Neuroscience, 31, 2792-2800.

Provine, R. R. (1989). Faces as releasers of contagious yawning: An approach to face detection using normal human subjects. Bulletin of the Psychonomic Society, 27, 211-214.

Puzzo, I., Cooper, N. R., Cantarella, S., \& Russo, R. (2011). Measuring the effects of manipulating stimulus presentation time on sensorimotor alpha and low beta reactivity during hand movement observation. NeuroImage, 57, 1358-1363.
Puzzo, I., Cooper, N. R., Vetter, P., \& Russo, R. (2010). EEG activation differences in the pre-motor cortex and supplementary motor area between normal individuals with high and low traits of autism. Brain Research, 1342, 104-110.

Ramachandran, V. S., \& Oberman, L. M. (2006). Broken mirrors: A theory of autism. Scientific American, 295, 62-69.

Rizzolatti, G., \& Craighero, L. (2004). The mirror-neuron system. Annual Review of Neuroscience, 27, 169-192.

Schippers, M. B., Roebroeck, A., Renken, R., Nanetti, L., \& Keysers, C. (2010). Mapping the information flow from one brain to another during gestural communication. Proceedings of the $\mathrm{Na}$ tional Academy of Sciences, 107, 9388-9393.

Schürmann, M., Hesse, M. D., Stephan, K. E., Saarela, M., Zilles, K., Hari, R., \& Fink, G. R. (2005). Yearning to yawn: The neural basis of contagious yawning. NeuroImage, 24, 1260-1264. doi:10.1016/j.neuroimage.2004.10.022

Senju, A., Kikuchi, Y., Akechi, H., Hasegawa, T., Tojo, Y., \& Osanai, H. (2009). Brief report: Does eye contact induce contagious yawning in children with autism spectrum disorder? Journal of Autism and Developmental Disorders, 39, 1598-1602.

Senju, A., Maeda, M., Kikuchi, Y., Hasegawa, T., Tojo, Y., \& Osanai, H. (2007). Absence of contagious yawning in children with autism spectrum disorder. Biology Letters, 3, 706-708.

Streltsova, A., Berchio, C., Gallese, V., \& Umiltà, M. A. (2010). Time course and specificity of sensory-motor alpha modulation during the observation of hand motor acts and gestures: A high density EEG study. Experimental Brain Research, 205, 363-373. doi:10.1007/s00221-010-2371-7 